\title{
Pacific
}

Journal of

Mathematics

\section{A MEAN VALUE INEQUALITY WITH APPLICATIONS TO BERGMAN SPACE OPERATORS}

\author{
PATRICK Robert Ahern and ZelJKo Cuckovic
}




\title{
A MEAN VALUE INEQUALITY WITH APPLICATIONS TO BERGMAN SPACE OPERATORS
}

\author{
PATRICK AHERN AND ŽELJKo ČUČKOVIĆ
}

\begin{abstract}
If $u$ is integrable over the unit disc and $u=T u$, where $T$ is the Berezin operator then it is known that $u$ must be harmonic. In this paper we give examples to show that the condition $T u \geq u$ does not imply that $u$ is subharmonic, but we are able to show that the condition $T u \geq u$ does imply that $u$ must be "almost" subharmonic near the boundary in an appropriate sense. We give two versions of this "almost" subharmonicity, a "pointwise" version and a "weak-star" version. We give applications of these results to hyponormal Toeplitz operators on the Bergman space.
\end{abstract}

\section{Introduction.}

Let $D$ be the open unit disc in the complex plane. We let $H^{\infty}(D)$ denote the space of bounded holomorphic functions in $D$ and let $B(D)$ denote the Bergman space on $D$; the set of holomorphic functions $f$ on $D$ such that

$$
\int_{D}|f(z)|^{2} d A(z)<\infty
$$

where $d A$ denotes planar Lebesgue measure on $D . B(D)$ is a closed subspace of the Hilbert space $L^{2}(d A)$ and so there is an orthogonal projection $P$ : $L^{2}(d A) \rightarrow B(D)$. If $\varphi \in L^{\infty}(d A)$ we define the Toeplitz operator $T_{\varphi}$ : $B(D) \rightarrow B(D)$ by $T_{\varphi} f=P(\varphi f)$. For each $z \in D$ we have the kernel function $k_{z}(\zeta)=\frac{1}{\pi(1-\bar{z} \zeta)^{2}}$. For each $f \in B(D)$ we have $f(z)=\left\langle f, k_{z}\right\rangle$ where $\langle f, g\rangle$ denotes the inner product in $L^{2}(d A)$. We use the usual notation of $\|f\|_{2}^{2}=$ $\langle f, f\rangle$ for $f \in L^{2}(d A)$. Note that $\left\|k_{z}\right\|_{2}^{2}=\left\langle k_{z}, k_{z}\right\rangle=k_{z}(z)=\frac{1}{\pi\left(1-|z|^{2}\right)^{2}}$. For each $z \in D$ we have the biholomorphic involution $\varphi_{z}: D \rightarrow D$ given by $\varphi_{z}(\zeta)=\frac{z-\zeta}{1-\bar{z} \zeta}$. With these involutions we can define the Berezin transform $T u$ of any $u \in L^{1}(d A)$, by

$$
T u(z)=\frac{1}{\pi} \int_{D} u \circ \varphi_{z} d A
$$


Equivalently, after a change of variables, we have

$$
T u(z)=\frac{\left(1-|z|^{2}\right)^{2}}{\pi} \int_{D} \frac{u(\zeta)}{|1-\bar{\zeta} z|^{4}} d A(\zeta)
$$

Finally, if $A$ is a bounded operator on a Hilbert space $X$, with norm $\|x\|$, we say $A$ is hyponormal if $A^{*} A \geq A A^{*}$, or in other words, if

$$
\|A x\| \geq\left\|A^{*} x\right\| \text { for all } x \in X .
$$

It is a simple matter to check that if $u$ is harmonic in $D$, i.e., $\Delta u(z)=$ $\partial^{2}$ $\frac{\partial}{\partial z \partial \bar{z}} u(z) \equiv 0$, and $u \in L^{1}(d A)$, then $T u(z)=u(z)$ for all $z \in D$. In [1], the converse was established, i.e., if $T u=u$ in $D$ then $u$ must be harmonic. Now if $u$ is subharmonic and in $L^{1}(d A)$ then it follows easily that $T u \geq u$ in $D$. We start Section 1 by showing the converse of this statement to be false, i.e., we show that there exists $u$ (indeed a large class of such $u$ ) so that $T u \geq u$ in $D$ but $u$ is not subharmonic. However in Theorem 2 we show that the condition $T u \geq u$ in $D$ implies some sort of vestigal subharmonicity near the boundary. We show, under a rather mild integrability condition on $\Delta u$, that if $T u \geq u$ in $D$ then $\varlimsup_{z \rightarrow \zeta} \Delta u(z) \geq 0$ for all $\zeta \in \partial D$. Actually Theorem 2 gives a more precise "local" theorem. The main tool in the proof is a formula that represents $T u-u$ as an integral of $\Delta u$ times a positive kernel. This is the content of Theorem 1.

Our second result of this type says that if $T u \geq u$ in $D$ and if the measures $\Delta u\left(r e^{i \theta}\right) d \theta$ have a weak-star limit as $r \rightarrow 1$ on some interval $I$, then that limit is a positive measure on $I$. This is Theorem 3 .

In the second section we give two applications of the results of the first section. In [2] H. Sadraoui showed that if $f, g \in H^{\infty}(D)$ and if $T_{f+\bar{g}}$ is hyponormal and if we assume that $f^{\prime}, g^{\prime}$ both lie in the Hardy class $H^{2}$, then $\left|f^{\prime}\left(e^{i \theta}\right)\right| \geq\left|g^{\prime}\left(e^{i \theta}\right)\right|$ a.e. on the unit circle. Our first result says that if $f, g \in H^{\infty}(D)$ and $T_{f+\bar{g}}$ is hyponormal, then $\varlimsup_{z \rightarrow e^{i \theta}}\left(\left|f^{\prime}(z)\right|-\left|g^{\prime}(z)\right|\right) \geq 0$ for all $e^{i \theta}$. Our second result says that if, in addition, there is an $\operatorname{arc} I$ on the circle such that $f^{\prime} \in H^{2}(I)$, (this is defined precisely in Section 2), then $g^{\prime}$ has the same property and $\left|f^{\prime}\left(e^{i \theta}\right)\right| \geq\left|g^{\prime}\left(e^{i \theta}\right)\right|$ a.e. on $I$. This last result can be viewed as a local version of Sadraoui's result and it contains his theorem as a special case.

\section{Section 1.}

We begin with an example of a function $u$ such that $T u \geq u$ in $D$ but $u$ is not subharmonic. Note that $\Lambda(a)=\int_{D}\left|\varphi_{a}\right| \frac{d A}{\pi}$ is continuous and $\Lambda(0)=2 / 3$ so there exists $\delta>0$ such that $\Lambda(a)>\frac{1}{2}$ if $|a|<\delta$. Now let $u$ be any 
strictly convex function that is continuous and integrable on $[0,1)$ such that $u(0)=u(\alpha)=0$ for some $0<\alpha<\frac{1}{2}$. Then we have $u(r)<0$ for $0<r<\alpha$ and $u$ has a minimum at a unique point $\beta, 0<\beta<\alpha$. We further assume that $\beta<\delta$. We regard $u$ as a radial function on $D$. We claim any such $u$ satisfies $T u \geq u$. First suppose $|a| \leq \beta$ then $u(a)=u(|a|)<0$. On the other hand

$$
\int\left|\varphi_{a}\right| \frac{d A}{\pi} \geq \frac{1}{2}>\alpha
$$

so

$$
0<u\left(\int\left|\varphi_{a}\right| \frac{d A}{\pi}\right) \leq \int u \circ \varphi_{a} \frac{d A}{\pi}
$$

the latter inequality is Jensen's. Hence

$$
u(a) \leq \int u \circ \varphi_{a} \frac{d A}{\pi}
$$

in this case.

If $|a|>\beta$ we have $a=\int \varphi_{a} \frac{d A}{\pi}$ and hence $|a| \leq \int\left|\varphi_{a}\right| \frac{d A}{\pi}$ and therefore

$$
u(a) \leq u\left(\int\left|\varphi_{a}\right| \frac{d A}{\pi}\right),
$$

because $u$ is strictly increasing on $(\beta, 1)$. Another application of Jensen's inequality proves that $u(a) \leq \int u \circ \varphi_{a} \frac{d A}{\pi}$ in this case. Clearly $u$ is not subharmonic since $u(0)=0$ and

$$
\frac{1}{2 \pi} \int_{0}^{2 \pi} u\left(r e^{i \theta}\right) d \theta=u(r)<0
$$

if $0<r<\alpha$.

Suppose $u \in C^{2}(D)$ and $0<r<1$, then starting from one of Green's identities we obtain the familiar formula

$$
u(0)=\frac{1}{2 \pi} \int_{0}^{2 \pi} u\left(r e^{i \theta}\right) d \theta+\frac{2}{\pi} \int_{|\zeta| \leq r} \Delta u(\zeta) \log \frac{|\zeta|}{r} d A(\zeta),
$$

which we may rewrite as

$$
\frac{1}{2 \pi} \int_{0}^{2 \pi} u\left(r e^{i \theta}\right) d \theta-u(0)=\frac{2}{\pi} \int_{|\zeta| \leq r} \Delta u(\zeta) \log \frac{r}{|\zeta|} d A(\zeta) .
$$

Next we multiply both sides of (2) by $2 r$ and integrate on $r$ from 0 to 1 . We obtain

$$
(T u)(0)-u(0)=\int_{|\zeta|<1} \Delta u(\zeta) K(\zeta) d A(\zeta)
$$


where

$$
K(\zeta)=\frac{4}{\pi} \int_{|\zeta|}^{1} r \log \frac{r}{|\zeta|} d r=\frac{1}{\pi}\left[\log \frac{1}{|\zeta|^{2}}-\left(1-|\zeta|^{2}\right)\right] .
$$

So far this is a purely formal calculation. To see what conditions are required on $u$, we look at the kernel $K$. We let $f(x)=\log \frac{1}{x}-(1-x)$, then an application of Taylor's formula with remainder shows that

$$
f(x)=\frac{1}{2 t^{2}}(x-1)^{2} \quad \text { where } 0<x<t<1 .
$$

From this we see that $f(x) \geq 0,0<x<1$ and

(6) $f(x) \geq \frac{1}{2}(1-x)^{2}$ for $0<x<1$, and $f(x) \leq 2(1-x)^{2}$ for $\frac{1}{2}<x<1$.

So (3) holds if $u \in C^{2}(D)$ and if

$$
\int_{|\zeta|<1}|u(\zeta)| d A(\zeta)<\infty \text { and } \int_{|\zeta|<1}|\Delta u(\zeta)|\left(1-|\zeta|^{2}\right)^{2} d A(\zeta)<\infty
$$

Now we wish to apply (3) not to $u$ but to $u \circ \varphi_{z}$. This yields

$$
T u(z)-u(z)=\int_{|\zeta|<1} \Delta\left(u \circ \varphi_{z}\right)(\zeta) K(\zeta) d A(\zeta) .
$$

Recalling that $\Delta\left(u \circ \varphi_{z}\right)(\zeta)=(\Delta u)\left(\varphi_{z}(\zeta)\right)\left|\varphi_{z}^{\prime}(\zeta)\right|^{2}$ and making the change of variables $\omega=\varphi_{z}(\zeta)$ we arrive at the following

Theorem 1. Suppose that $u \in C^{2}(D)$ and that

$$
\int_{|\zeta|<1}|u(\zeta)| d A(\zeta)<\infty
$$

and

$$
\int_{|\zeta|<1}|\Delta u(\zeta)|\left(1-|\zeta|^{2}\right)^{2} d A(\zeta)<\infty
$$

Then

$$
T u(z)-u(z)=\int_{|\zeta|<1} \Delta u(\zeta) K(z, \zeta) d A(\zeta)
$$

where

$$
K(z, \zeta)=\frac{1}{\pi}\left[\log \frac{1}{\left|\varphi_{z}(\zeta)\right|^{2}}-\left(1-\left|\varphi_{z}(\zeta)\right|^{2}\right)\right]
$$


Moreover the kernel $K$ satisfies:

$$
K(z, \zeta) \geq \frac{1}{2 \pi}\left[\frac{\left(1-|z|^{2}\right)\left(1-|\zeta|^{2}\right)}{|1-\bar{z} \zeta|^{2}}\right]^{2} \quad \text { for } z, \zeta \in D
$$

and

$$
\begin{gathered}
K(z, \zeta) \leq \frac{2}{\pi}\left[\frac{\left(1-|z|^{2}\right)\left(1-|\zeta|^{2}\right)}{|1-\bar{z} \zeta|^{2}}\right]^{2} \quad \text { if } \\
\frac{\left(1-|z|^{2}\right)\left(1-|\zeta|^{2}\right)}{|1-\bar{z} \zeta|^{2}}<\frac{1}{2}
\end{gathered}
$$

Proof. Everything has been proved except (7) and (8) but they follow from (6) and the well-known identity

$$
1-\left|\varphi_{z}(\zeta)\right|^{2}=\frac{\left(1-|z|^{2}\right)\left(1-|\zeta|^{2}\right)}{|1-\bar{z} \zeta|^{2}}
$$

The following well-known estimate is proved by a straightforward calculation that we omit.

Lemma 1. There exists a constant $C_{0}>0$ such that

$$
\int_{|\zeta|<1} \frac{\left(1-|\zeta|^{2}\right)^{2}}{|1-\bar{z} \zeta|^{4}} d A(\zeta) \geq C_{0} \log \frac{1}{1-|z|}
$$

Theorem 2. Suppose that $u \in C^{2}(D)$,

$$
\begin{aligned}
& \int_{|\zeta|<1}|u(\zeta)| d A(\zeta)<\infty, \\
& \int_{|\zeta|<1}|\Delta u(\zeta)|\left(1-|\zeta|^{2}\right)^{2} d A(\zeta)<\infty,
\end{aligned}
$$

and that $\varlimsup_{z \rightarrow \zeta_{0}} \Delta u(z)<0$ for some $\zeta_{0} \in \partial D$. Then there exists $\delta>0$ such that $T u(z)<u(z)$ for all $z \in D$ such that $\left|z-\zeta_{0}\right|<\delta$.

Proof. For convenience we assume that $\zeta_{0}=1$. By assumption there exists $a>0$ and $\epsilon>0$ such that if $z \in D$ and $|z-1|<\epsilon$, then $\Delta u(z) \leq-a$. If $D(1, \epsilon)$ denotes the set of points in $D$ with $|z-1|<\epsilon$ and $D(1, \epsilon)^{\prime}$ the complement of $D(1, \epsilon)$ in $D$, then we have

$$
\begin{aligned}
\int_{D} \Delta u(\zeta) K(z, \zeta) d A(\zeta)= & \int_{D(1, \epsilon)} \Delta u(\zeta) K(z, \zeta) d A(\zeta) \\
& +\int_{D(1, \epsilon)^{\prime}} \Delta u(\zeta) K(z, \zeta) d A(\zeta)
\end{aligned}
$$


We deal with the second integral: if $|z-1|<\epsilon / 2$ and $\zeta \in D(1, \epsilon)^{\prime}$, then $|1-\bar{\zeta} z|$ is bounded away from 0 and hence

$$
\frac{\left(1-|z|^{2}\right)\left(1-|\zeta|^{2}\right)}{|1-\bar{\zeta} z|^{2}} \leq C\left(1-|z|^{2}\right)<1 / 2
$$

if $1-|z|^{2}$ is sufficiently small, and hence by (8) we have $K(z, \zeta) \leq C(1-$ $\left.|z|^{2}\right)^{2}\left(1-|\zeta|^{2}\right)^{2}$, so

$\left|\int_{D(1, \epsilon)^{\prime}} \Delta u(\zeta) K(z, \zeta) d A(\zeta)\right| \leq C\left(1-|z|^{2}\right)^{2} \int_{D(1, \epsilon)^{\prime}}|\Delta u(\zeta)|\left(1-|\zeta|^{2}\right)^{2} d A(\zeta)$.

Note that this is $O\left(\left(1-|z|^{2}\right)^{2}\right)$. Next

$$
\begin{aligned}
\int_{D(1, \epsilon)} \Delta u(\zeta) K(z, \zeta) d A(\zeta) \leq & -a \int_{D(1, \epsilon)} K(z, \zeta) d A(\zeta) \\
= & -a \int_{D} K(z, \zeta) d A(\zeta)+a \int_{D(1, \epsilon)^{\prime}} K(z, \zeta) d A(\zeta) \\
\leq & -\frac{a}{2 \pi} \int_{D} \frac{\left(1-|z|^{2}\right)^{2}\left(1-|\zeta|^{2}\right)^{2}}{|1-\bar{\zeta} z|^{4}} d A(\zeta) \\
& +\frac{2 a}{\pi} \int_{D(1, \epsilon)^{\prime}} \frac{\left(1-|z|^{2}\right)^{2}\left(1-|\zeta|^{2}\right)^{2}}{|1-\bar{\zeta} z|^{4}} d A(\zeta) \\
\leq & -C_{0} a\left(1-|z|^{2}\right)^{2} \log \frac{1}{1-|z|}+O\left(\left(1-|z|^{2}\right)^{2}\right)
\end{aligned}
$$

Here we have used (7) and (8) again as well as Lemma 1. Combining these estimates we have, for $|1-z|<\epsilon / 2$,

$$
T u(z)-u(z) \leq-C_{0} a\left(1-|z|^{2}\right)^{2} \log \frac{1}{1-|z|}+O\left(\left(1-|z|^{2}\right)^{2}\right),
$$

which becomes negative as $z$ approaches 1 .

The next lemma shows that the inequality $T u \geq u$ is preserved under certain convolutions.

Lemma 2. Suppose $u \in L^{1}(D)$ and $T u \geq u$ in $D$. Suppose $w \geq 0$ is a bounded measurable function on the circle. Define, for $z \in D$,

$$
U(z)=\frac{1}{2 \pi} \int_{0}^{2 \pi} u\left(z e^{-i t}\right) w\left(e^{i t}\right) d t .
$$


Then $U \in L^{1}(D)$ and

$$
T U \geq U \text { in } D
$$

Proof. Note that if $z=r e^{i \theta}$, then

$$
\begin{aligned}
U\left(r e^{i \theta}\right) & =\frac{1}{2 \pi} \int_{0}^{2 \pi} u\left(r e^{i(\theta-t)}\right) w\left(e^{i t}\right) d t \\
& =\frac{1}{2 \pi} \int_{0}^{2 \pi} u\left(r e^{i t}\right) w\left(e^{i(\theta-t)}\right) d t
\end{aligned}
$$

By hypothesis,

$$
u\left(r e^{i t}\right) \leq \frac{\left(1-r^{2}\right)^{2}}{\pi} \int_{0}^{1} \rho \int_{0}^{2 \pi} \frac{u\left(\rho e^{i(t-s)}\right)}{\left|1-r \rho e^{i s}\right|^{4}} d s d \rho .
$$

Since $w \geq 0$ we can multiply both sides of this inequality by $w\left(e^{i(\theta-t)}\right)$ and integrate on $t$. After interchanging the order of integration we get

$$
\begin{aligned}
U\left(r e^{i \theta}\right) & =\frac{1}{2 \pi} \int_{0}^{2 \pi} u\left(r e^{i t}\right) w\left(e^{i(\theta-t)}\right) d t \\
& \leq \frac{\left(1-r^{2}\right)^{2}}{\pi} \int_{0}^{1} \rho \int_{0}^{2 \pi} \frac{1}{\left|1-r \rho e^{i s}\right|^{4}} \int_{0}^{2 \pi} u\left(\rho e^{i(t-s)}\right) w\left(e^{i(\theta-t)}\right) \frac{d t}{2 \pi} d s d \rho \\
& =\frac{\left(1-r^{2}\right)^{2}}{\pi} \int_{0}^{1} \rho \int_{0}^{2 \pi} \frac{1}{\left|1-r \rho e^{i s}\right|^{4}} \int_{0}^{2 \pi} u\left(\rho e^{i t}\right) w\left(e^{i(\theta-t-s)}\right) \frac{d t}{2 \pi} d s d \rho \\
& =\frac{\left(1-r^{2}\right)^{2}}{\pi} \int_{0}^{1} \rho \int_{0}^{2 \pi} \frac{1}{\left|1-r \rho e^{i s}\right|^{4}} U\left(\rho e^{i(\theta-s)}\right) d s d \rho \\
& =(T U)\left(r e^{i \theta}\right) .
\end{aligned}
$$

The next theorem says that if $T u \geq u$ and $\Delta u$ has a weak* limit on some interval, that limit is non-negative.

Theorem 3. Suppose that $u \in C^{2}(D) \cap L^{1}(D)$, and that $\int_{D}|\Delta u(\zeta)|(1-$ $\left.|\zeta|^{2}\right)^{2} d A(\zeta)<\infty$. Suppose further that $T u \geq u$ in $D$ and that there is a closed arc $I$ on the boundary of the unit circle and a finite Borel measure $\mu$ on I such that for all continuous functions $\varphi$ on I we have

$$
\lim _{r \rightarrow 1} \int_{I} \Delta u\left(r e^{i \theta}\right) \varphi\left(e^{i \theta}\right) \frac{d \theta}{2 \pi}=\int_{I} \varphi d \mu,
$$

then $\mu \geq 0$ on $\stackrel{\circ}{I}$, the interior of $I$.

Proof. Let $w\left(e^{-i t}\right)$ be a continuous non-negative function with compact support in $\stackrel{\circ}{I}$, let

$$
U(z)=\frac{1}{2 \pi} \int_{0}^{2 \pi} u\left(z e^{-i t}\right) w\left(e^{i t}\right) d t
$$


From Lemma 2 we know that $T U \geq U$ in $D$. Since the Laplacian commutes with rotations it follows from (9) that

$$
\Delta U(z)=\frac{1}{2 \pi} \int_{0}^{2 \pi}(\Delta u)\left(z e^{-i t}\right) w\left(e^{i t}\right) d t
$$

and hence that

$$
\int_{D}|\Delta U(\zeta)|\left(1-|\zeta|^{2}\right)^{2} d A(\zeta)<\infty
$$

It follows from Theorem 2 that there exists $r_{k} \rightarrow 1$ and $\theta_{k} \rightarrow 0$ such that $\lim _{k \rightarrow \infty} \Delta U\left(r_{k} e^{i \theta_{k}}\right) \geq 0$. Now it follows from (10) that

$$
\Delta U\left(r_{k} e^{i \theta_{k}}\right)=\frac{1}{2 \pi} \int_{0}^{2 \pi} \Delta u\left(r_{k} e^{i t}\right) w\left(e^{i\left(\theta_{k}-t\right)}\right) d t .
$$

Notice that for all $k$ sufficiently large $w\left(e^{i\left(\theta_{k}-t\right)}\right)$ will have its support in $\stackrel{\circ}{I}$. We have

$$
\begin{aligned}
& \int_{I} w\left(e^{-i t}\right) d \mu(t)-\Delta U\left(r_{k} e^{i \theta_{k}}\right) \\
& =\int_{I} w\left(e^{-i t}\right) d \mu(t)-\int_{I} \Delta u\left(r_{k} e^{i t}\right) w\left(e^{-i t}\right) \frac{d t}{2 \pi} \\
& \quad+\int_{I} \Delta u\left(r_{k} e^{i t}\right)\left[w\left(e^{-i t}\right)-w\left(e^{i\left(\theta_{k}-t\right)}\right)\right] \frac{d t}{2 \pi} .
\end{aligned}
$$

The first difference above goes to 0 as $r_{k} \rightarrow 1$ by hypothesis. The second difference is bounded in modulus by

$$
\left(\sup _{k} \int_{I}\left|\Delta u\left(r_{k} e^{i t}\right)\right| \frac{d t}{2 \pi}\right)\left(\sup _{t}\left|w\left(e^{-i t}\right)-w\left(e^{i\left(\theta_{k}-t\right)}\right)\right|\right) .
$$

The first factor is bounded, by the principle of uniform boundedness and the second goes to zero as $k \rightarrow \infty$ by the uniform continuity of $w$. We have shown that $\int_{I} w\left(e^{-i t}\right) d \mu(t) \geq 0$ for all non-negative $w\left(e^{-i t}\right)$ continuous with compact support in $\stackrel{\circ}{I}$; the result follows.

\section{Section 2.}

Now suppose that $f$ and $g$ are holomorphic in $D$ and $f+\bar{g}=\varphi$ is bounded. We wish to calculate $\left\|T_{\varphi} F\right\|_{2}^{2}$ for $F \in H^{\infty}(D)$

$$
T_{\varphi} F=P(f+\bar{g}) F=f F+P(\bar{g} F)
$$


so

$$
\begin{aligned}
\left\|T_{\varphi} F\right\|_{2}^{2} & =\langle f F+P(\bar{g} F), f F+P(\bar{g} F)\rangle \\
& =\|f F\|_{2}^{2}+\|P \bar{g} F\|_{2}^{2}+\langle P \bar{g} F, f F\rangle+\langle f F, P \bar{g} F\rangle \\
& =\|f F\|_{2}^{2}+\|P \bar{g} F\|_{2}^{2}+\langle\bar{f} \bar{g} F, F\rangle+\langle f g F, F\rangle,
\end{aligned}
$$

since $P$ is self-adjoint.

By interchanging the roles of $f$ and $g$ we see that

$$
\left\|T_{\bar{\varphi}} F\right\|_{2}^{2}=\|g F\|_{2}^{2}+\langle\bar{f} \bar{g} F, F\rangle+\langle f g F, F\rangle+\|P \bar{f} F\|_{2}^{2} .
$$

Hence $T_{\varphi}$ is hyponormal if and only if

$$
\|f F\|_{2}^{2}+\|P \bar{g} F\|_{2}^{2} \geq\|g F\|_{2}^{2}+\|P \bar{f} F\|_{2}^{2}
$$

for all $F \in H^{\infty}(D)$.

In particular (9) holds if $F=k_{z}$ for some $z \in D$. Now it is immediate that $\bar{g} k_{z}-\overline{g(z)} k_{z} \perp B(D)$ for any $g \in H^{\infty}(D)$ and hence that $P\left(\bar{g} k_{z}\right)=\overline{g(z)} k_{z}$.

Theorem 4. Suppose that $f$ and $g$ are holomorphic in $D$, that $f+\bar{g}=\varphi$ is bounded in $D$ and that $T_{\varphi}$ is hyponormal, then $T u \geq u$ in $D$ where $u(z)=$ $|f(z)|^{2}-|g(z)|^{2}$.

Proof. By the above discussion, if we let $F=k_{z}$ in (9) we get

$$
\left\|f k_{z}\right\|_{2}^{2}+|g(z)|^{2}\left\|k_{z}\right\|_{2}^{2} \geq\left\|g k_{z}\right\|_{2}^{2}+|f(z)|^{2}\left\|k_{z}\right\|_{2}^{2} .
$$

Since $\left\|k_{z}\right\|_{2}^{2}=\frac{1}{\pi\left(1-|z|^{2}\right)^{2}}$, a minor rearrangement of (10) proves the theorem.

Corollary. Suppose that $f$ and $g$ are holomorphic in $D$, that $f+\bar{g}=\varphi$ is bounded in $D$ and that $T_{\varphi}$ is hyponormal, then $\overline{\lim }_{z \rightarrow \zeta}\left(\left|f^{\prime}(z)\right|^{2}-\left|g^{\prime}(z)\right|^{2}\right) \geq 0$ for every $\zeta \in \partial D$. In particular, if $f^{\prime}$ and $g^{\prime}$ are continuous at $\zeta \in \partial D$, then $\left|f^{\prime}(\zeta)\right| \geq\left|g^{\prime}(\zeta)\right|$.

Proof. The proof follows from the theorem and the simple observation that $\Delta|f|^{2}=\left|f^{\prime}\right|^{2}$ for any holomorphic $f$.

Suppose that $f$ is holomorphic in an open set of the form

$$
\left\{r e^{i \theta}: r_{0}<r<1 \text { and } e^{i \theta} \in I\right\}
$$


where $I$ is some open arc on the boundary of the unit circle. We say that $f \in H^{2}(I)$ if

(i) $f$ has polynomial growth i.e., there exists $A>0$ such that $f\left(r e^{i \theta}\right)=$ $O\left((1-r)^{-A}\right)$ for all $e^{i \theta} \in I$.

(ii) There exists $r_{k} \rightarrow 1$ such that

$$
\int_{I}\left|f\left(r_{k} e^{i \theta}\right)\right|^{2} d \theta \leq C<\infty, \quad \text { all } k .
$$

The next lemma is standard. Since we know of no convenient references we indicate the proof.

Lemma 3. Suppose $f \in H^{2}(I)$, then there exists $F \in L^{2}(I)$ such that $\lim _{r \rightarrow 1} f\left(r e^{i \theta}\right)=F\left(e^{i \theta}\right)$ a.e. on $I$ and for every compact subinterval $J \subset I$

$$
\lim _{r \rightarrow 1} \int_{J}\left|f\left(r e^{i \theta}\right)-F\left(e^{i \theta}\right)\right|^{2} d \theta=0 .
$$

In particular, $\varlimsup_{r \rightarrow 1} \int_{J}\left|f\left(r e^{i \theta}\right)\right|^{2} d \theta<\infty$.

Proof. Pick a compact interval $L$ such that $J \subseteq \stackrel{\circ}{L} \subseteq L \subseteq I$. Let $e^{i \theta_{1}}, e^{i \theta_{2}}$ be the end points of $L$ and choose $N$ such that

$$
\lim _{r \rightarrow 1}\left[\left(r e^{i \theta}-e^{i \theta_{1}}\right)\left(r e^{i \theta}-e^{i \theta_{2}}\right)\right]^{N} f\left(r e^{i \theta}\right)=0
$$

if $\theta=\theta_{1}$ or $\theta_{2}$. This is possible by i). Let $g(z)=\left[\left(z-e^{i \theta_{1}}\right)\left(z-e^{i \theta_{2}}\right)\right]^{N} f(z)$. Let $r_{0}<r_{1}<1$ and $\Delta_{k}=\left\{r e^{i \theta}: r_{1} \leq r \leq r_{k}, e^{i \theta} \in L\right\}$. Let $\partial \Delta_{k}=\Gamma_{k} \cup L_{k}$ where $L_{k}=\left\{r_{k} e^{i \theta}: e^{i \theta} \in L\right\}$. If $z \in \stackrel{\circ}{k}_{k}$ we have

$$
g(z)=\frac{1}{2 \pi i} \int_{\Gamma_{k}} \frac{g(\zeta)}{\zeta-z} d \zeta+\frac{1}{2 \pi i} \int_{L_{k}} \frac{g(\zeta)}{\zeta-z} d \zeta
$$

If we let $k \rightarrow \infty$ we get $g(z)=g_{1}(z)+g_{2}(z)$ where $g_{1}(z)$ is holomorphic on $\stackrel{\circ}{L}$ and $g_{2}(z)$ is the Cauchy integral of an $L^{2}$ function on the circle. It follows that the conclusions of the lemma hold for $g$ and hence for $f$.

Theorem 5. Suppose that $f$ and $g$ are holomorphic in $D$, that $f+\bar{g}=\varphi$ is bounded in $D$ and that $T_{\varphi}$ is hyponormal. Suppose further that there is an open interval $I$ such that $f^{\prime} \in H^{2}(I)$. Then for any open subinterval $J \subseteq \bar{J} \subseteq I g^{\prime} \in H^{2}(J)$ and $\left|f^{\prime}\left(e^{i \theta}\right)\right| \geq\left|g^{\prime}\left(e^{i \theta}\right)\right|$ almost everywhere on $I$.

Proof. Let $w\left(e^{-i t}\right)$ be a continuous function with compact support in $I$ such that $0 \leq w \leq 1$ and $w\left(e^{-i t}\right) \equiv 1$ on a neighborhood of $\bar{J}$, combining Theorems 2 and 3 with Lemma 2 we have the existence of $r_{k} \rightarrow 1$ and $\theta_{k} \rightarrow 0$ 
such that

$$
\lim _{k \rightarrow \infty} \int_{0}^{2 \pi}\left(\left|f^{\prime}\left(r_{k} e^{i t}\right)\right|^{2}-\left|g^{\prime}\left(r_{k} e^{i t}\right)\right|^{2}\right) w\left(e^{i\left(\theta_{k}-t\right)}\right) d t \geq 0
$$

Let $L$ be compact interval so that $\bar{J} \subseteq \stackrel{\circ}{L} \subseteq L \subseteq I$. As before, for large $k$, $w\left(e^{i\left(\theta_{k}-t\right)}\right)$ has support in $L$ and hence,

$$
\begin{aligned}
& \int_{0}^{2 \pi}\left|f^{\prime}\left(r_{k} e^{i t}\right)\right|^{2} w\left(e^{i\left(\theta_{k}-t\right)}\right) d t \\
& \quad \leq \int_{L}\left|f^{\prime}\left(r_{k} e^{i t}\right)\right|^{2} d t \leq C<\infty, \quad \text { by Lemma } 3 .
\end{aligned}
$$

Also, for large $k, w\left(e^{i\left(\theta_{k}-t\right)}\right) \equiv 1$ on $J$ from which it follows that

$$
\varliminf_{k \rightarrow \infty} \int_{J}\left|g^{\prime}\left(r_{k} e^{i t}\right)\right|^{2} d t \leq C<\infty .
$$

Now since $g \in H^{\infty}(D), g^{\prime}$ has polynomial growth and hence $g^{\prime} \in H^{2}(J)$. It now follows that the measures $\left(\left|f^{\prime}\left(r e^{i \theta}\right)\right|^{2}-\left|g^{\prime}\left(r e^{i \theta}\right)\right|^{2}\right) \frac{d \theta}{2 \pi}$ have a weak * limit as $r \rightarrow 1, e^{i \theta} \in J$, and that this limit is $\left(\left|f^{\prime}\left(e^{i \theta}\right)\right|^{2}-\left|g^{\prime}\left(e^{i \theta}\right)\right|^{2}\right) \frac{d \theta}{2 \pi}$. It follows that $\left|f^{\prime}\left(e^{i \theta}\right)\right| \geq\left|g^{\prime}\left(e^{i \theta}\right)\right|$ a.e. on $J$, and hence on $I$ since $J \subseteq \bar{J} \subseteq I$, was arbitrary.

\section{References}

[1] P. Ahern, M. Flores and W. Rudin, An invariant volume-mean-value property, Journal of Functional Analysis, vol. 111, 2 (1993), 380-397.

[2] H. Sadraoui, Hyponormal Toeplitz operators on the Bergman space, preprint.

Received October 12, 1993.

UNIVERSITY OF WISCONSIN-MADISON

MADISON, WI 53706

E-mail address: ahern@math.wisc.edu

AND

UNIVERSITY OF TOLEDO

Toledo, OH 43606

E-mail address: zcuckovi@math.utoledo.edu 

Peng Lin and Richard Rochberg, Trace ideal criteria for Toeplitz and Hankel operators on the weighted Bergman spaces with exponential type

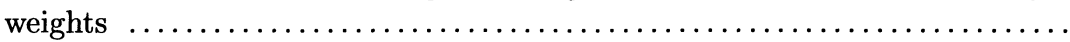

Donald E. Marshall and Arne Stray, Interpolating Blaschke products . .

Kathy D. Merrill and Lynne $\mathbf{H}$. Walling, On quadratic reciprocity over

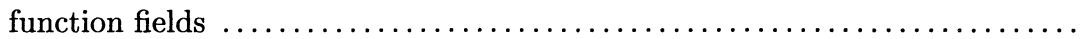

Takahiko Nakazi and Masahiro Yamada, $\left(A_{2}\right)$-conditions and Carleson

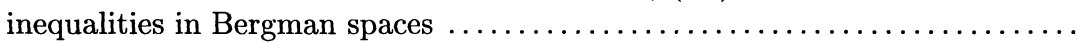

C. Ott, A note on a paper of E. Boasso and A. Larotonda ..............

Victor Patrangenaru, Classifying 3 and 4 dimensional homogeneous Riemannian manifolds by Cartan triples

Carlo Pensavalle and Tim Steger, Tensor products with anisotropic principal series representations of free groups

Ying Shen, On Ricci deformation of a Riemannian metric on manifold with boundary

Albert Jeu-Liang Sheu, The Weyl quantization of Poisson $S U(2) \ldots \ldots$

Alexandra Shlapentokh, Polynomials with a given discriminant over fields of algebraic functions of positive characteristic

Eric Stade and D.I. Wallace, Weyl's law for $S L(3, \mathbb{Z}) \backslash S L(3, \mathbb{R}) / S O(3, \mathbb{R})$

Christopher W. Stark, Resolutions modeled on ternary trees ........ 557

Per Tomter, Minimal hyperspheres in two-point homogeneous spaces .....

Jun Tomiyama, Topological Full groups and structure of normalizers in

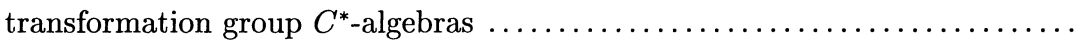

Nik Weaver, Subalgebras of little Lipschitz algebras 


\section{PACIFIC JOURNAL OF MATHEMATICS}

\section{Volume $173 \quad$ No. $2 \quad$ April 1996}

A mean value inequality with applications to Bergman space operators

PATRICK ROBERT AHERn and ZELJKO CUCKOVIC

$H^{p}$-estimates of holomorphic division formulas

MATS ANDERSSON and HASSE CARLSSON

Group structure and maximal division for cubic recursions with a double root

Christian JeAn-Claude Ballot

The Weil representation and Gauss sums

ANTONiA WiLson BLUHER

Duality for the quantum $E(2)$ group

ALFONS VAN DAELE and S. L. WORONOWICZ

Cohomology complex projective space with degree one codimension-two fixed submanifolds 387

KARL HEINZ DOVERMANN and ROBERT D. LITTLE

On the mapping intersection problem

ALEXANDER DRANISHNIKOV

From the $L^{1}$ norms of the complex heat kernels to a Hörmander multiplier theorem for

sub-Laplacians on nilpotent Lie groups

\section{XUAN THINH DUONG}

Isoperimetric inequalities for automorphism groups of free groups

Allen E. Hatcher and Karen Vogtmann

Approximation by normal elements with finite spectra in $C^{*}$-algebras of real rank zero

HUAXIN LIN

Interpolating Blaschke products

DonALD EdDY MARShall and ARNE STRAY

Interpolating Blaschke products generate $H^{\infty}$

JOHN BRADY GARNETT and ARTUR NICOLAU

Classifying 3- and 4-dimensional homogeneous Riemannian manifolds by Cartan triples

VICTOR PATRANGENARU

Polynomials with a given discriminant over fields of algebraic functions of positive

characteristic

ALEXANDRA SHLAPENTOKH

Resolutions modeled on ternary trees

CHRISTOPHER W. STARK

Topological full groups and structure of normalizers in transformation group $C^{*}$-algebras 